\title{
Integrating Phosphoproteomics and Bioinformatics to Study Brassinosteroid-Regulated Phosphorylation Dynamics in Arabidopsis
}

Li-Ling Lin ${ }^{1 \dagger}$, Chia-Lang Hsu' ${ }^{1 \dagger}$, Chia-Wei Hu², Shiao-Yun Ko ${ }^{2}$, Hsu-Liang Hsieh ${ }^{3}$, Hsuan-Cheng Huang ${ }^{4 *}$ and Hsueh-Fen Juan ${ }^{1,2,5^{*}}$

\begin{abstract}
Background: Protein phosphorylation regulated by plant hormone is involved in the coordination of fundamental plant development. Brassinosteroids (BRs), a group of phytohormones, regulated phosphorylation dynamics remains to be delineated in plants. In this study, we performed a mass spectrometry (MS)-based phosphoproteomics to conduct a global and dynamic phosphoproteome profiling across five time points of BR treatment in the period between 5 min and $12 \mathrm{~h}$. MS coupling with phosphopeptide enrichment techniques has become the powerful tool for profiling protein phosphorylation. However, MS-based methods tend to have data consistency and coverage issues. To address these issues, bioinformatics approaches were used to complement the non-detected proteins and recover the dynamics of phosphorylation events.
\end{abstract}

Results: A total of 1104 unique phosphorylated peptides from 739 unique phosphoproteins were identified. The time-dependent gene ontology $(G O)$ analysis shows the transition of biological processes from signaling transduction to morphogenesis and stress response. The protein-protein interaction analysis found that most of identified phosphoproteins have strongly connections with known BR signaling components. The analysis by using Motif-X was performed to identify 15 enriched motifs, 11 of which correspond to 6 known kinase families. To uncover the dynamic activities of kinases, the enriched motifs were combined with phosphorylation profiles and revealed that the substrates of casein kinase 2 and mitogen-activated protein kinase were significantly phosphorylated and dephosphorylated at initial time of BR treatment, respectively. The time-dependent kinase-substrate interaction networks were constructed and showed many substrates are the downstream of other signals, such as auxin and ABA signaling. While comparing BR responsive phosphoproteome and gene expression data, we found most of phosphorylation changes were not led by gene expression changes. Our results suggested many downstream proteins of BR signaling are induced by phosphorylation via various kinases, not through transcriptional regulation.

Conclusions: Through a large-scale dynamic profile of phosphoproteome coupled with bioinformatics, a complicated kinase-centered network related to BR-regulated growth was deciphered. The phosphoproteins and phosphosites identified in our study provide a useful dataset for revealing signaling networks of BR regulation, and also expanded our knowledge of protein phosphorylation modification in plants as well as further deal to solve the plant growth problems.

Keywords: Phosphoproteomics, Bioinformatics, Brassinosteroids, Phosphorylation dynamics, Kinase-centered network

\footnotetext{
* Correspondence: hsuancheng@ym.edu.tw; yukijuan@ntu.edu.tw

†Equal contributors

${ }^{4}$ Institute of Biomedical Informatics, Center for Systems and Synthetic

Biology, National Yang-Ming University, No.155, Sec.2, Linong Street, Taipei

112, Taiwan

'Department of Life Science, National Taiwan University, No. 1, Sec. 4,

Roosevelt Road, Taipei 106, Taiwan

Full list of author information is available at the end of the article
}

\section{Biomed Central}

(c) 2015 Lin et al. This is an Open Access article distributed under the terms of the Creative Commons Attribution License (http://creativecommons.org/licenses/by/4.0), which permits unrestricted use, distribution, and reproduction in any medium, provided the original work is properly credited. The Creative Commons Public Domain Dedication waiver (http:// creativecommons.org/publicdomain/zero/1.0/) applies to the data made available in this article, unless otherwise stated. 


\section{Background}

Brassinosteroids (BRs) are steroid plant hormones essential for normal plant growth and development. Numerous plant processes are regulated by BRs, including cell elongation and division, photosynthesis, photomorphogenesis, flowering, seed germination, root development and abiotic/biotic stress response [1-5]. Therefore, understanding the response mechanism of BR regulation is critical for promoting agricultural development and boosting crop productivity by improving plant growth conditions.

Extensive studies using genetic and molecular approaches have identified major BR signaling components, which indicate that protein phosphorylation plays a crucial role in $B R$ regulations $[3,6,7]$. The cell surface receptor complex containing the receptor-like kinase, BR-insensitive 1 (BRI1), responds to BR signaling components by inducing the initial phosphorylation-dependent signal transduction cascade [3]. The binding of BR to the extracellular domain of BRI1 leads to auto-phosphorylation of BRI1 which phosphorylate BRI1 KINASE INHIBITOR 1 (BKI1), and then induces trans-phosphorylation between BRI1 and BRI1-associated receptor kinase1 (BAK1). Activated BRI1 phosphorylates BR-signaling kinases (BSKs) and BRinsensitive 2 (BIN2) which can transduce its signals to BRI1 suppressor1 (BSU1) phosphatase [6], and then stops phosphorylation of Brassinazole-resistant1 (BZR1)/BRI1ems-suppressor 1 (BES1) to induce BR-responsive target gene expression [8]. In negatively regulated stomatal cell formation, the activation of BIN2 could be reduced by BR, to inhibit mitogen-activated protein kinase kinase 4, 5, 7 and 9 (MKK4/5/7/9), which induce the phosphorylation of mitogen-activated protein kinase 3 and 6 (MPK3/6) [9]. In the immune response, BAK1 phosphorylation interacts as a co-receptor with BRI1-induced BR signaling and flagellin-sensing 2 (FLS2) signaling to increase disease resistance [10]. In brief, BSKs activate downstream phosphorylation signals to result in expression of BR target genes and to regulate stomatal formation, innate immunity, and stress responses during plant development.

Several proteomics studies also identified many proteins which are post-translationally modified in response to BR [11-14]. Huang and colleagues used two-dimensional (2-D) gel electrophoresis to investigate the regulatory relationship between BR and chilling condition in mug been epicotyl and found BR-induced proteins are involved in methionine assimilation, ATP synthesis, cell wall construction and the stress response [11]. Deng and colleagues identified posttranslational modification of BiP2, a luminal binding protein under BR treatment using 2-D difference gel electrophoresis (DIGE) [12]. Similarly, another study combining prefractionation with 2-D DIGE in Arabidopsis identified phosphorylation sites of BZR1, two tetratricopeptide repeat proteins, a phosphoenolpyruvate carboxykinase (PCK1), and a novel BR-induced plasma membrane protein
(DREPP) which are associated with BR promotion of plant growth [13]. Shigeta and colleagues identified several BRresponsive proteins located in the nucleus, including nucleosome assembly protein $1 ; 1$ (NAP1;1), NAP1;2, band 7 family protein, vernalization independence 3 , sadenosylmethionine synthetase 2 , and $60 \mathrm{~S}$ ribosomal protein L14, and found that NAP1;2 has a posttranslational modification in response to cellular BR levels [14]. Consequently, a comprehensive identification of phosphorylation events under the stimulus of BR is necessary to uncover the BR-regulated signaling networks.

Recent advances in phosphoproteomics, including highaccuracy mass spectrometry (MS) and phosphopeptideenrichment techniques, have allowed identification of previously nearly undetectable site-specific phosphorylations in plants [15]. Current methods for enriching phosphopeptides prominently include strong cation exchange (SCX) chromatography, immobilized metalaffinity chromatography (IMAC), and titanium dioxide $\left(\mathrm{TiO}_{2}\right)$ chromatography. SCX separates phosphopeptides from non-phosphopeptides by their solution charge state, but is unsuitable as a stand-alone method [16]. IMAC and $\mathrm{TiO}_{2}$ affinity chromatography are commonly used for phosphoproteome enrichment because of their high affinity for phosphopeptides. However, conventional IMAC suffers from partial non-specific absorption resulting from acidic non-phosphopeptides, though it can be improved by methyl esterification [17, 18]. Recently, the method of aliphatic hydroxy acidmodified metal oxide chromatography (HAMMOC) was invented to substantially improve the efficiency and specificity of $\mathrm{TiO}_{2}$ phosphopeptides enrichment [19]. It can reduce the interaction between non-phosphopeptides and metal oxides, thus removing most non-phosphopeptides from samples.

Although several studies have profiled the protein expression under BR response by MS techniques [11-14, 20-22], the dynamics of protein phosphorylation in response to $B R$ induction is not comprehensively identified till now. To capture the dynamics of phosphorylation events under BR regulatory responses, we performed a time-dependent study in Arabidopsis cell suspension cultures with 24epibrassinolide (a highly active BR). Dimethyl labeling and HAMMOC combined with nano-liquid-chromatographytandem mass spectrometry (nanoLC-MS/MS) was used to characterize the phosphoproteome of BR signaling processes.

However, because MS-based proteomics tends to have data consistency (poor reproducibility and inter-sample agreement) [23] and coverage (inability to detect the entire proteome) [24] issues, it is difficult to profile all phosphorylation sites at whole time points. Several technical approaches, such as exhaustive fractionation of samples [25] and repeated MS runs of the same samples [26], are widely 
used to address these issues, but these are still unable to completely overcome the problems. Recent studies have used bioinformatics approaches, such as biological networks [27], to complement the existing experimental approaches to increase the comprehensiveness of proteome coverage and enhance analytical resolution. Therefore, we used several bioinformatics approaches, including enrichment analysis as well as construction of protein-protein and kinase-substrate interaction networks to reveal the phosphorylation dynamics of BR in Arabidopsis.

\section{Results}

To determine appropriate BR treatment concentrations in Arabidopsis cells

Selection of suitable material for phosphoproteomic analysis was important for the investigation of the molecular mechanism of BR. PSB-D cells were chosen because of their short culturing time and growth condition characteristics, similar to those of root cells. To determine the most suitable concentration of exogenous BR24-epibrassinolide for cell treatment, we evaluated the activation of BR signaling in Arabidopsis cells based on the expression levels of two genes, small auxin up RNA (SAUR-AC1) [28] and indole-3-acetic acid amido synthetase (BRU6) [29], known to be upregulated by BR. Because previous studies have shown that the expression of SAUR-AC1 and BRU6 in five-day-old Arabidopsis cells were apparently changed after the treatment of BR for $3 \mathrm{~h}$ [30]. We found that SAUR-AC1 and BRU6 were clearly upregulated at concentrations of 1 and $5 \mu \mathrm{M}$ (Fig. 1a), whereas higher expression of SAUR-AC1 compared with BRU6 was observed at $5 \mu \mathrm{M}$ of 24-epibrassinolide which is also the same as used in previous Arabidopsis cells studies [31, 32]. Therefore, we used $5 \mu \mathrm{M}$ of 24-epibrassinolide for the subsequent experiments.

\section{Characterization of the BR-regulated phosphoproteome in Arabidopsis cells}

To accurately determine the BR-regulated phosphoproteins in Arabidopsis, we performed a time-course phosphorylation experiment and applied a quantitative phosphoproteomic analysis approach (Fig. 1b). We collected samples from cells treated with $5 \mu \mathrm{M}$ BR or mock at five time points: $5 \mathrm{~min}, 30 \mathrm{~min}, 3 \mathrm{~h}, 6 \mathrm{~h}$, and $12 \mathrm{~h}$. A total of 1104 unique phosphopeptides were identified, derived from 739 distinct phosphoproteins at a peptide FDR of $<1 \%$. Most of the identified phosphopeptides contained either one or two phosphorylation sites (Fig. 2a). Among all identified phosphorylation sites (Additional file 1), 1231 were considered as highconfidence hits (class I, phosphorylation localization probability $>0.75)$. Additionally, 249 phosphorylation sites were classified as class II $(0.5<p \leq 0.75)$ and 158 as class III $(p \leq 0.5)$. In further analysis, only class I sites among 644 proteins were used. A total of 858 serines (Ser), 259 threonines (Thr), and 114 tyrosines (Tyr) were identified as high-confidence phosphorylation sites, yielding a Ser/Thr/Tyr phosphorylation ratio of 69.7/ 21.0/9.3 \% (Fig. 2b). The number of identified phosphopeptides/phosphoproteins was similar among different time points (Fig. 2c and Table 1).

Since MS-based proteomics have consistency and coverage issues, 693 out of 1231 high-confident phosphorylation sites were assigned a ratios at least one time point, but only 394 out of 1231 phosphorylation sites were quantified at all five time points (Additional file 2). Although these phosphorylation sites contained missing values, they might be still informative for understanding the dynamics of BR signaling via bioinformatics approaches. Therefore, a total of 1087 quantitative phosphorylation sites were considered for further analysis.

\section{Biological processes of Arabidopsis cells mediated by BR dynamic phosphosignaling}

To obtain functional insights into the biological processes and cellular organization dynamically affected by BR treatment, Gene Ontology (GO) enrichment analysis was conducted. A threshold of 2-fold phosphorylation change (e.g. $\mid \log _{2}$ (fold-change) $\mid \geqq 1$ ) was applied to reveal 129 positively and 135 negatively BR-induced phosphorylation sites on 171 distinct proteins (Fig. 3a), and GO enrichment analysis was performed on these proteins. The proteins regulated at initial time points (BR treatment for $5 \mathrm{~min}$ ) significantly localized on plasma membrane (Fig. 3b) and enriched on the biological process term "BR mediated signaling pathway" (Fig. 3c and Additional file 3). Indeed, BR signaling is initiated from a series of transmembrane receptors and receptor-like kinases which are located in plasma membrane [7]. With the increasing time of BR treatment, we observed the propagation of BR effects. In the annotation of cellular component ontology, phosphorylated proteins enriched from 5 min samples are mostly plasma membrane proteins, and enriched from $30 \mathrm{~min}$ samples are mostly cytosol and nucleus proteins (Fig. 3b). Interestingly, the phosphorylated proteins after $3 \mathrm{~h}$ were still enriched on cytosol compared with 6 and $12 \mathrm{~h}$ treatment data. We speculated that these proteins may be the BR-responsive genes in transcriptional level and activated or suppressed by phosphorylation. For example, acyl-CoA binding protein 4 (ACBP4), tubulin alpha-1 chain (TUA1) and tubulin alpha-4 chain (TUA4) located in cytosol are BR-responsive genes [6] and have also significantly phosphorylation change at $3 \mathrm{~h}$. The similar trend was also observed in the annotation of biological process ontology (Fig. 3c). In addition to the biological process term "BR mediated signaling pathway", phosphorylated proteins at 5 min also involved in "proton transport" and "cellulose metabolic process" which are likely to contribute to BR 


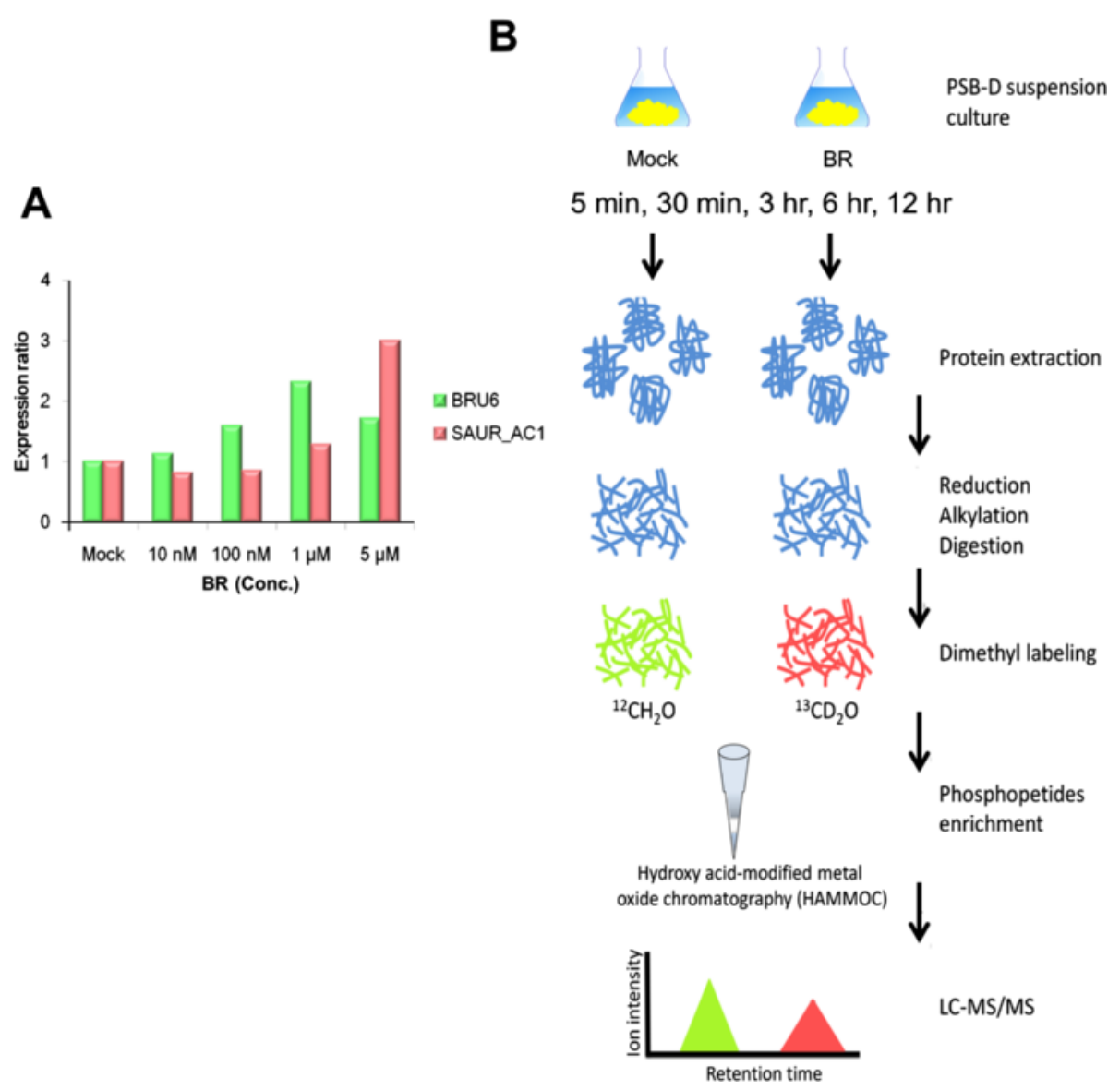

Fig. 1 Phosphoproteomic study strategy. a To determine appropriate treatment concentrations, two BR-regulated genes of the expression level (BRU6 and SAUR-AC1) were detected in different BR concentrations using qRT-PCR. The expression ratio shows gene expression of different BR concentrations against mock-treated RNA. The indicated concentrations were used for a PSB-D cell suspension culture for 3 h. 185 ribosomal RNA was assigned to the reference gene. b For phosphoproteomic study, PSB-D cells were treated with BR for 5 min, $30 \mathrm{~min}, 3 \mathrm{~h}, 6 \mathrm{~h}$, and $12 \mathrm{~h}$. Peptide dimethylation and titanium dioxide phosphopeptide enrichment were combined with nano-liquid chromatography-tandem mass spectrometry (nanoLC-MS/MS) analysis to characterize the phosphoproteome of the BR signaling process

promotion of solute uptake and directional cell elongation [2]. At $30 \mathrm{~min}$, phosphorylated proteins could be contributed to diverse processes, such as cell growth and proliferation, morphogenesis, and RNA processing (Fig. 3c). This might indicate that the signal delivers to nucleus for transcription after less than 30 min of BR treatment. These results of functional analyses from temporal phosphoproteomic experimental display the dynamic of BR signaling.

To clarify that these enriched biological processes are uniquely involved by BR-induced phosphoproteins, we compared the enriched GO terms using the BRresponsive phosphoproteins and genes derived from genome-wide transcriptional experiments [6, 33, 34]. A total of 4326 genes obtained from Wang and colleagues [6] show altered expression levels by BR treatment or by both bri1 and bzr1 mutations. After GO enrichment analysis, $532 \mathrm{GO}$ biological process terms were enriched in the BR-responsive genes (adjusted
$P$-value $<0.05)$. After comparing two sets of enriched GO terms, 98 GO terms were uniquely enriched on BRinduced phosphorylated proteins (Fig. 3c and Additional file 3). These unique processes include "gluconeogenesis", "response to gravity", "RNA silencing", "RNA transport", "chromosome segregation", and "protein glycosylation". These results suggest that these processes are regulated through proteins whose phosphorylation sites are induced by BR.

\section{Protein-protein interaction network unveils the possible BR-regulated network}

To better understand the relationships among all identified phosphoproteins, we constructed a protein-protein interaction network (PIN) derived from BioGRID [35], AtPID [36], and PhosPhAt [37]. Additionally, PhosPhAt also provides the known kinase-substrate interactions. The constructed PIN was composed of 99 proteins, including 18 BR-related proteins collected from literature 

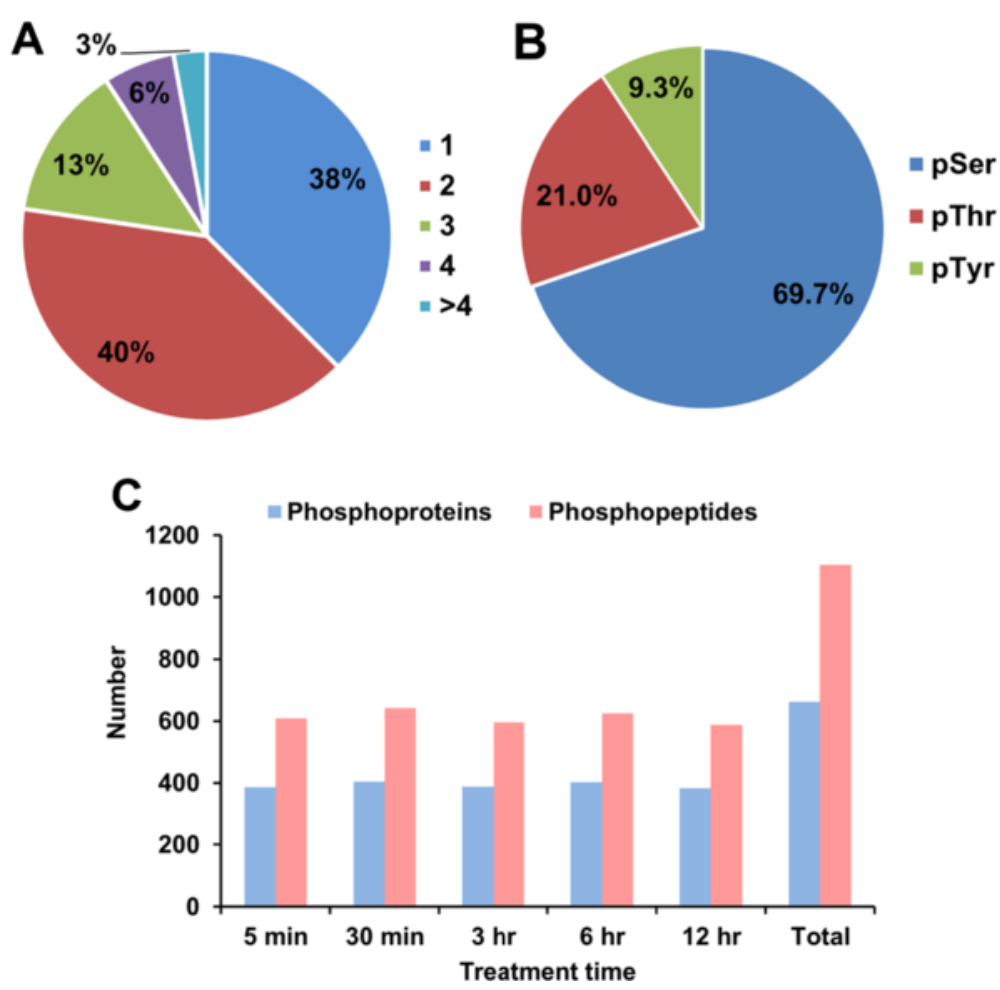

Fig. 2 Characteristics of phosphorylated residues, phosphopeptides, and phosphoproteins data obtained in the study. a Frequency of phosphorylated residues distributed in the phosphopeptides: one phosphorylation site is $38 \%$, two phosphorylation sites is $40 \%$, three phosphorylation sites is $13 \%$, four phosphorylation sites is $6 \%$ and more than four phosphorylation sites is $3 \%$. b Distribution of phosphorylation sites (class I) in the phosphoproteomic data. c Number of unique phosphopeptides and phosphoproteins identified in each time point experiment

$[3,6,7]$ and 81 phosphoproteins identified in this study, and 144 interactions (Fig. 4). From the network, we found that many phosphoproteins identified in this study were known or strongly associated with core components of BR signaling. Several known BR signaling components were also identified in our experiment, including brassinosteroid-signaling kinase 3 (BSK3) [6], GSK3/Shaggy-like protein kinase 1 (GSK1) [38], MPK3/MPK6 [9, 39, 40], 14-3-3-like protein G-box factor 14 phi (GF14 PHI) [41], and BSU 1-like 1 and 3 (BSL1 and BSL3) [38].

In addition to these known BR signaling components, many phosphoproteins might be the BR signaling associated proteins according to the PIN results (Fig. 4). Besides BSK3, a group of receptor-like cytoplasmic kinases (RLCK) II family with the consensus phosphosites to BSK3, including AT5G59010, AT3G54030, and AT5G41260, interacts with upstream components of BR signals such as BRI1, BSK1, BSK3 and BIN2. These proteins were significantly up-regulated at $5 \mathrm{~min}$, indicating that they may play the roles as co-regulated kinases in the initial steps of BR signaling. SHAGGY-related kinase 11 (SK11), SHAGGYrelated protein kinase 12 (AtSK12), and SHAGGY-like kinase 13 (SK13) belonging to subgroup I of Arabidopsis GSK3/SHAGGY-like kinases are important in BR signaling by phosphorylating BZR1 [38]. Moreover, many proteins are the substrates of mitogen-activated protein kinase (MPK3, MPK4 and MPK6), and regulated at the late time points; it indicates that BR signalling could control divergent processes through the mitogen activated protein kinase (MAPK) pathway [9].

Table 1 The number of phosphorylation sites in the time-course analysis

\begin{tabular}{llllll}
\hline & $5 \mathrm{~min}$ & $30 \mathrm{~min}$ & $3 \mathrm{~h}$ & $6 \mathrm{~h}$ & $12 \mathrm{~h}$ \\
\hline pS & $550(78.4 \%)$ & $604(79.0 \%)$ & $564(77.8 \%)$ & $592(79.8 \%)$ & $560(80.8 \%)$ \\
pT & $116(16.5 \%)$ & $120(15.7 \%)$ & $116(16.0 \%)$ & $108(14.6 \%)$ & $101(14.6 \%)$ \\
pY & $36(5.1 \%)$ & $41(5.4 \%)$ & $45(6.2 \%)$ & $42(5.7 \%)$ & $32(4.6 \%)$ \\
total & 702 & 765 & 724 & 742 & 693 \\
\hline
\end{tabular}


A

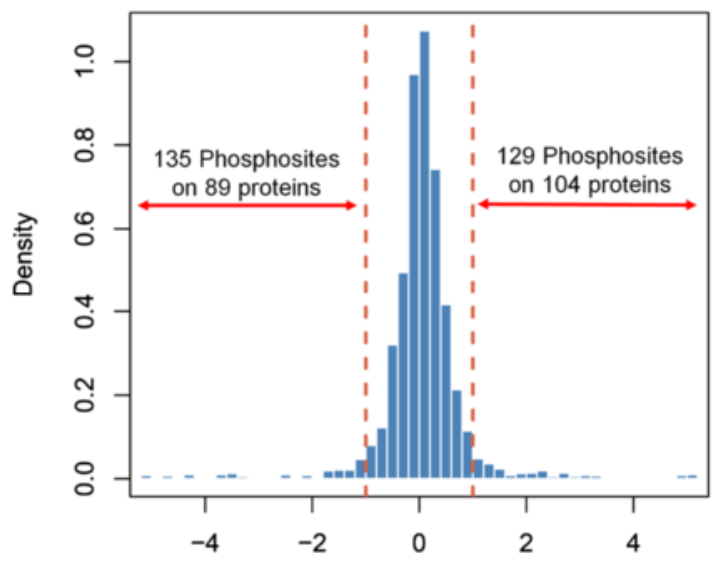

B

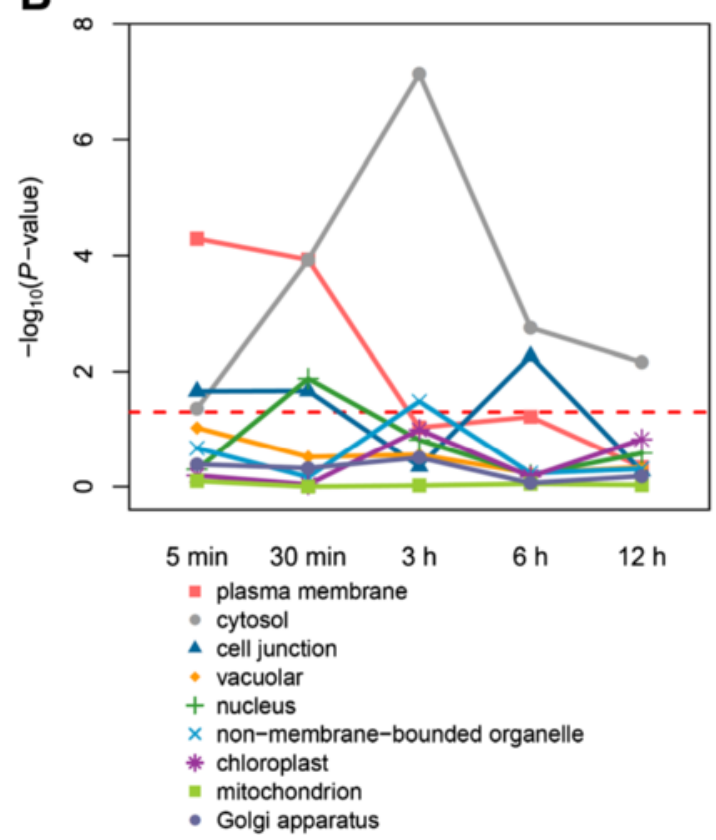

C

Organic substance metabolic process

Ion transport \& homeostatic process

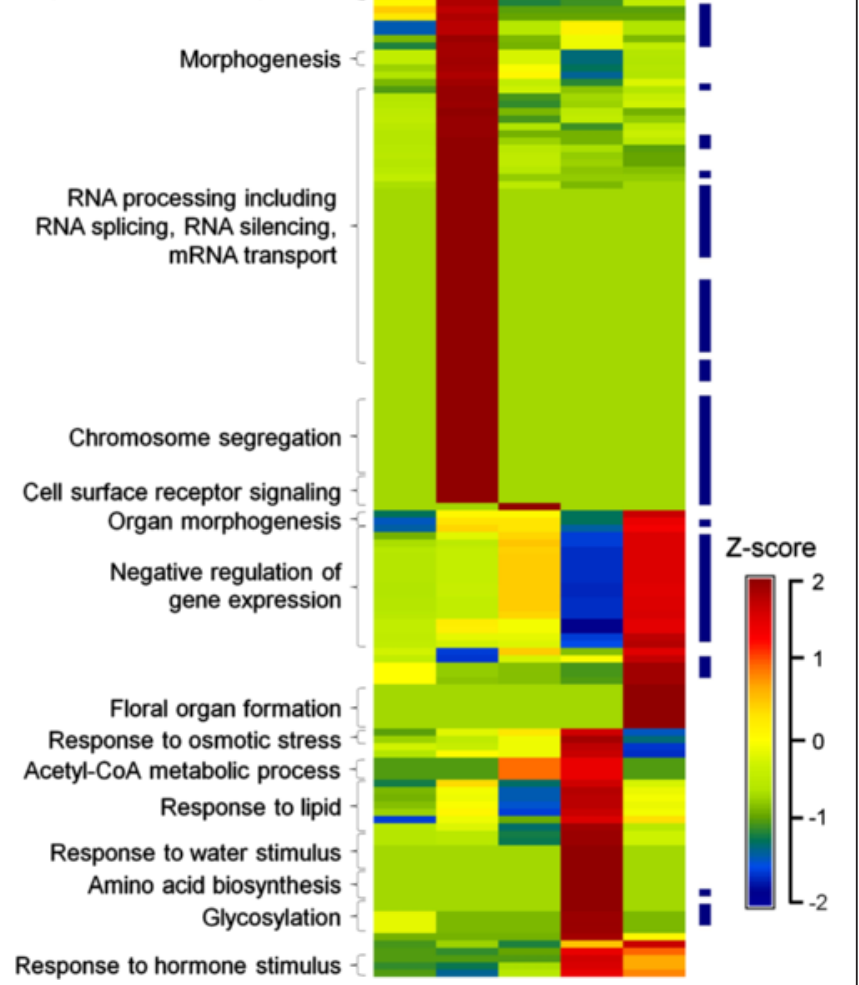

Fig. 3 Functional groups in significantly regulated phosphoproteins. a Distribution of all quantified phosphopeptides in BR treatment and BR-responsive sites which has two-fold phosphorylation change. $\mathbf{b}$ The proteins with BR-responsive sites are annotated according to cellular compartment GO terms. The $P$-value is obtained from Fisher's exact test with Benjamini-Hochberg correction. The red dash line denotes the $P$-value $=0.05$. $\mathbf{c}$ The heatmap presents the dynamic change of biological process with similar analysis as in (b). The values in the heatmap represent the z-transformation of $-\log _{10}(P$-value) by GO term (Color scales: red, high overrepresentation; blue, high underrepresentation). The unique GO terms are lighted in dark blue

\section{Various kinases involved in the BR-transduced downstream phosphosignals}

The sequence consensus of phosphopeptide motifs reflects the kinase-specific regulation of substrates and the identification of the corresponding kinases. To find primary initial protein phosphorylation inducers, we submitted the flanking sequences of high-confident phosphorylation residues to Motif-X. After motif analysis, 13 serine and 2 threonine significantly enriched motifs were identified, but no tyrosine motif was significantly enriched (Table 2). Through literature and database survey [37, 42, 43], some motifs corresponding kinases are well-known: pSDDE, pS[D/ $\mathrm{E}] \mathrm{X}[\mathrm{D} / \mathrm{E}]$ and $\mathrm{p}[\mathrm{S} / \mathrm{T}] \mathrm{XX}[\mathrm{D} / \mathrm{E}]$ for casein kinase II (CK2) family; $\mathrm{p}[\mathrm{S} / \mathrm{T}] \mathrm{P}$ for GSK-3, cyclin dependent kinase (CDK) and MAPK families; RXXpS for SNF1-related kinase II (SnRK2) family; pSXXS for casein kinase I (CK1) family. Although no kinases in plant can be assigned to DpS, EpS, and pSXP, they are similar to the recognized motifs of some kinases in human (Additional file 4): DpS for large tumor suppressor kinase 1 (LAST1) and NIMA-related 


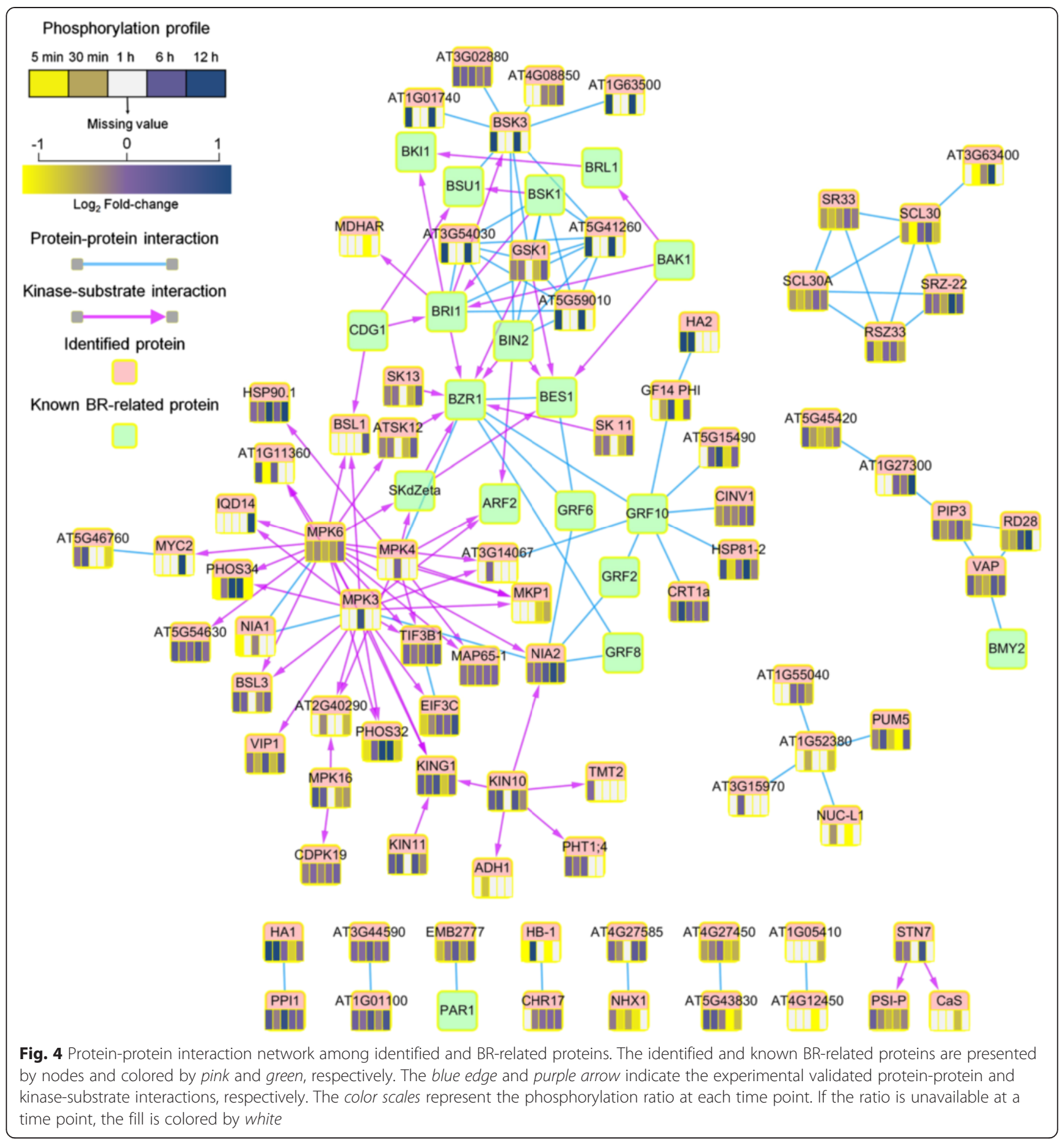

kinase 2 (NEK2), EpS for pre-mRNA processing factor 4B (PRPF4B), and pSXP for casein kinase 1, gamma 3 (CSNK1G3), dual-specificity tyrosine-phosphorylation regulated kinase 4 (DYRK4), and PDLIM1 interacting kinase 1 like (PDIK1L). The motif, pSXDXE, has not yet been assigned to any specific kinases, it may be novel and interesting for further investigation.

Sequentially, we combined motifs with phosphorylation profiles to reveal the dynamics of $\mathrm{BR}$ signaling transduction (Fig. 5). Although only few motifs were considered as significant enrichment, i.e. $-\log _{10}(P$-value) $\geqq 1.3$, at some time points, these analyses are useful to observe the activation profile of a specific kinase after BR treatment. The phosphorylation levels of proteins containing one of MAPK recognizing motifs pTP tend to be down-regulated in the initial steps and then upregulated at the later time points. This phosphorylation trend is also observed in PIN where most of the 
Table 2 The enriched motifs of all identified phosphopeptides

\begin{tabular}{|c|c|c|c|}
\hline Motif & Corresponding kinase & $\begin{array}{l}\text { No. of } \\
\text { occurrence }\end{array}$ & $\begin{array}{l}\text { No. phosphorylation } \\
\text { change (two-fold) }\end{array}$ \\
\hline pSDDE & CK2 & 32 & 5 \\
\hline pSDXE & CK2 & 99 & 16 \\
\hline pSDXD & CK2 & 73 & 5 \\
\hline pSP & GSK3, MAPK, CDK & 139 & 24 \\
\hline pSEXE & CK2 & 58 & 7 \\
\hline pSXDXE & Unknown & 41 & 3 \\
\hline Dps & $\begin{array}{l}\text { Similar to human kinases: } \\
\text { LAST1 and NEK2 }\end{array}$ & 141 & 20 \\
\hline EpS & $\begin{array}{l}\text { Similar to human } \\
\text { kinase: PRPF4B }\end{array}$ & 133 & 23 \\
\hline RXXpS & SnRK2, CaMK & 75 & 18 \\
\hline pSXP & $\begin{array}{l}\text { Similar to human kinases: } \\
\text { CSNK1G3, DYRK4, } \\
\text { and PDIK1L }\end{array}$ & 76 & 14 \\
\hline pSXXD & CK2 & 147 & 14 \\
\hline pSXXE & CK2 & 233 & 28 \\
\hline pSXXS & CK1 & 107 & 21 \\
\hline pTP & MAPK, CDK & 34 & 9 \\
\hline PTXXE & CK2 & 36 & 7 \\
\hline
\end{tabular}

${ }^{a}$ No. of occurrence on sites with two-fold phosphorylation change at any time point

substrates of MPK3/MPK6 were up-regulated at 6 or $12 \mathrm{~h}$ (Fig. 4), and consistent with the previous study in which MAPK may initially be suppressed by BRregulated GSK3/Shaggy-like kinases [9]. Moreover, the phosphosites with motif pTXXE was increasingly phosphorylated at $5 \mathrm{~min}$ and $30 \mathrm{~min}$, but those with motif pSDDE and pSEXE/pSXXE were decreasingly phosphorylated at $30 \mathrm{~min}$ and $3 \mathrm{~h}$, respectively. The result suggests their corresponding kinase, CK2, plays a crucial role in BR signaling. Indeed, CK2 is important for cell growth and cell viability, and may make crosstalk between $B R$ and other signaling, such as auxin and abscisic acid (ABA) signalling [44, 45]. In addition, SnRK2, a central component of ABA signaling, of which substrates (RXXpS) were increasingly phosphorylated at $3 \mathrm{~h}$, suggesting the interaction between $\mathrm{BR}$ and $\mathrm{ABA}$ signaling pathways [46]. Among these motifs, the phosphosites with motif pSXP were decreasingly phosphorylated at $30 \mathrm{~min}$. Although the corresponding kinase of motif pSXP in Arabidopsis is still unknown, previous study has also observed that this motif is enriched in the upregulated phosphopeptides under osmotic stress [47].

\section{A dynamic phosphorylation network in BR-regulated Arabidopsis cells}

On the basis of the results presented above, the relation between kinases and substrates were determined by identification of the enriched motifs at phosphorylation sites differential with more than two-fold. We focused on the transition of phosphorylation events from $5 \mathrm{~min}$ to $30 \mathrm{~min}$ and constructed time-dependent kinasesubstrate networks for 5 and $30 \mathrm{~min}$, respectively (Fig. 6). Several proteins have been demonstrated associated with the response of BR signaling. The phosphorylation of $\mathrm{H}(+)$-ATPases (HAs), including HA1 and HA2, were persistently activated by CK2 during 5 and $30 \mathrm{~min}$ treatment. Indeed, the activation of $\mathrm{H}(+)$-ATPase is necessary for the maintenance of homeostasis due to BR inductions of hyperpolarization and cell wall expansion [48]. Similarly, ATP-binding cassette sub-family B member 4 (ABCB4) was positively phosphorylated at 30 min treatment and may assist auxin transport for BR response [33]. This might also explain the persistent activation of auxinresponsive GH3-like protein (AT1G48660) after BR treatment (Additional file 1). Another interesting result, the phosphorylation of BZR-insensitive-long hypocotyls 4

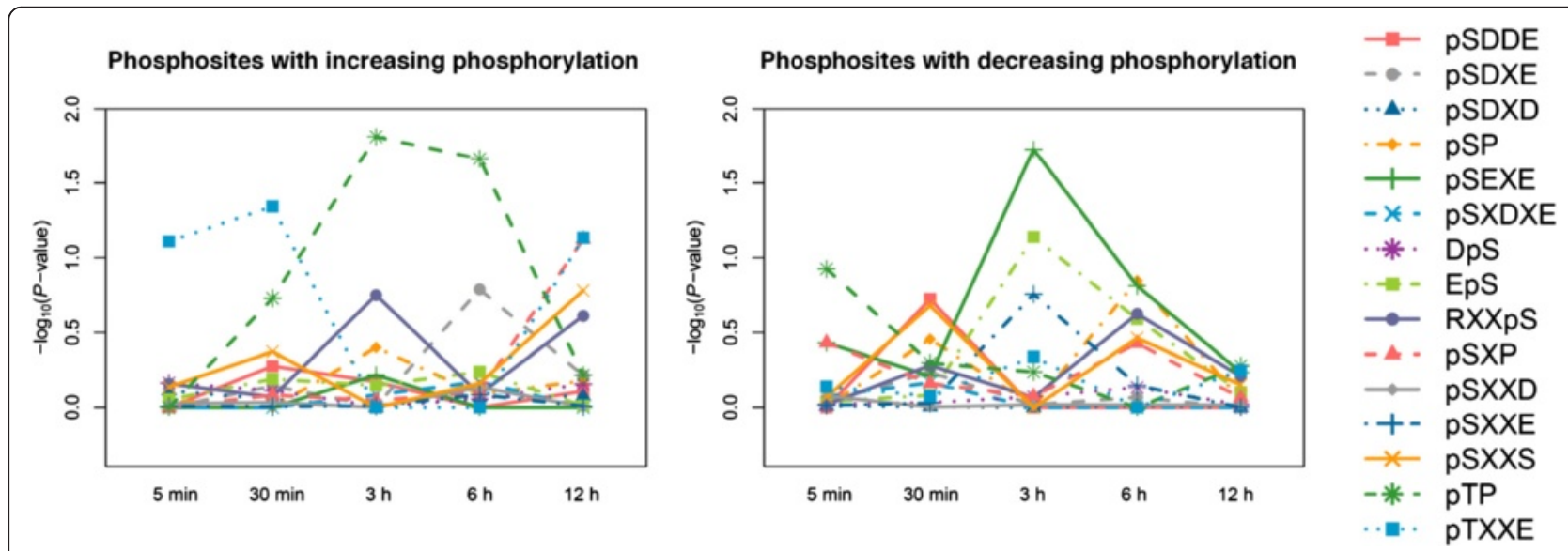

Fig. 5 The time-dependent phosphorylation trend of substrates of kinase motifs. The P-value is obtained by Fisher's exact test and used to assess whether phosphosites with increasing (left figure) and decreasing phosphorylation (right figure) under BR treatment compared to control are enriched on a certain motif of a given time point 


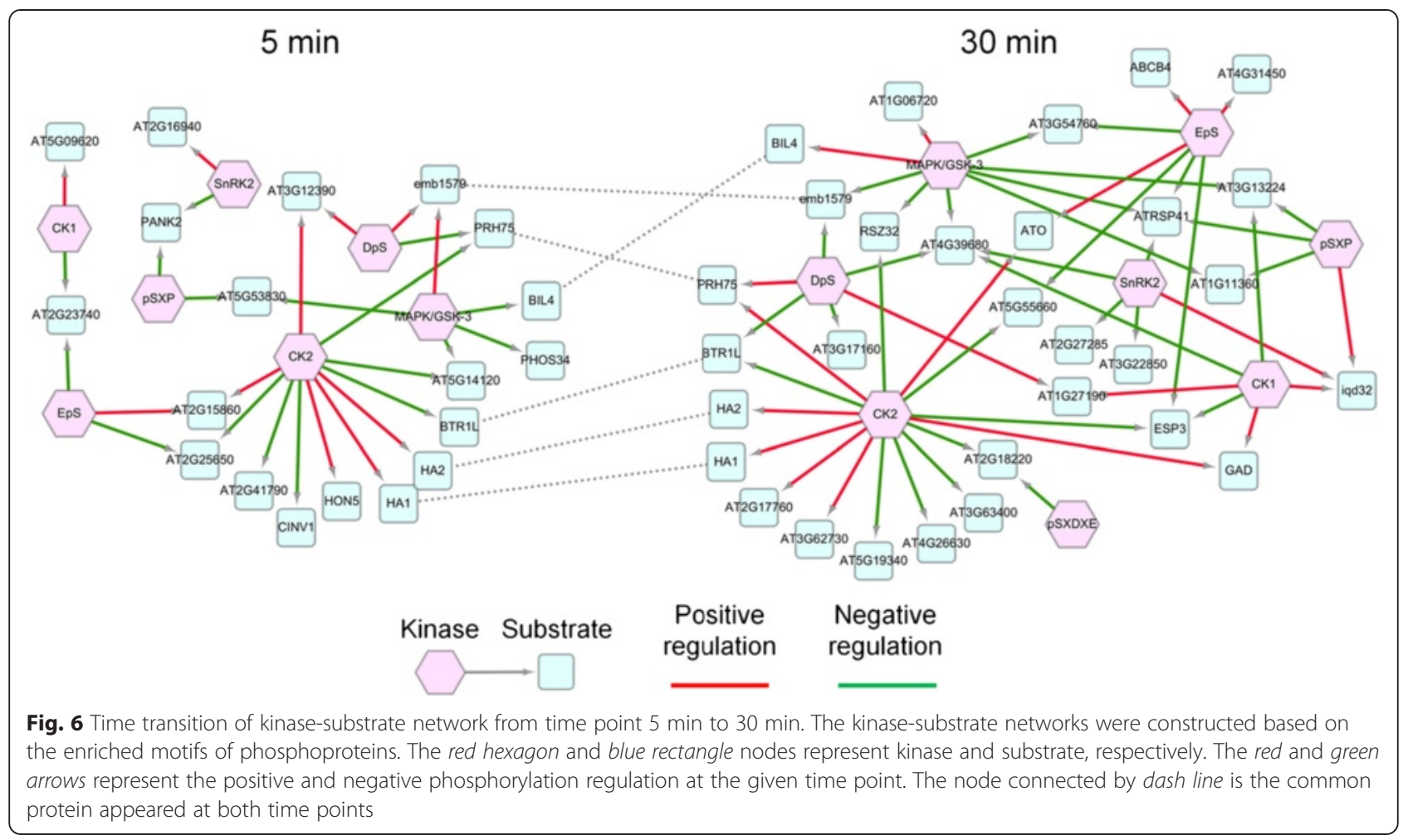

(BIL4) was down-regulated at 5 min treatment and then up-regulated at $30 \mathrm{~min}$ by MAPK. This observation is consistent with the previous study that BIL4 acts as a slow effective factor for mediating cell elongation in BR signalling [49]. Leucine-rich repeat protein kinase family protein (AT1G27190), RING/U-box superfamily protein (AT4G31450), and hypothetical protein (AT5G19340) were significantly regulated at $30 \mathrm{~min}$ treatment by different kinases. Although the function of these proteins is still unclear, all of them reveal altered expression levels after BR treatment [33, 34]. Additionally, AT5G19340 is the BZR1 directly regulated gene [33]. IQ-domain 32 (Iqd32), a proteins containing IQ-domain, was up-regulated at 30 min treatment. It could facilitate cellular RNA localization as one mechanism to control and fine-tune gene expression and protein sorting for certain types of cellular signalling [50]. In the kinase-substrate network at $30 \mathrm{~min}$ treatment, several proteins have been reported that can be phosphorylated by ABA regulation, such as arginine/serine-rich-splicing factor 41 (ATRSP41), binding to Tomato mosaic virus RNA 1 Long form (BTR1L), serine/arginine-rich splicing factor Z32 (RSZ32; AT3G53500), iqd32, SAP domain-containing protein (AT4G39680), DEK domain-containing chromatin associated protein (AT4G26630), and adenine nucleotide alpha hydrolases-like protein (AT1G11360) [51]. To sum up, this constructed dynamic phosphorylation network can provide the possible downstream events of BRinduced signaling.

\section{Comparison of BR-induced phosphorylated proteins and expressed genes}

Brassinosteroid induces not only phosphorylation change for signaling transduction, but also expression change for responding comprehensively biological processes. Therefore, the identified phosphorylation change may have a bias due to the expression change on transcriptional levels. To clarify this concern, we compared the identified phosphoproteins with the 4326 BR-responsive genes as previously described. 102 out of 644 identified phosphoproteins are also BR-responsive genes, including $55 \mathrm{BR}$ induced genes, 46 BR-repressed genes, and one gene that show complex expression in different experiment (Additional file 5). However, these two data were not significantly overlapped ( $P$-value $=0.50$, Fisher's exact test) (Fig. 7a). If only considering the proteins with significantly phosphorylation changes, there were 33 proteins and the overlap is also not statistically significant $(P$-value $=0.066$, Fisher's exact test $)$. In these 33 common proteins, the gene expression and phosphorylation regulation trend in most of them are consistent, but six proteins are not (Additional file 5). Furthermore, we examined whether the proteins with significant phosphorylation changes were enriched in BR-responsive gene for each time point. We found only significantly regulated proteins at $6 \mathrm{~h}$ were enriched in BR-responsive genes $(P$-value $=0.011$, Fisher's exact test) (Fig. 7b). These results reveal that the phosphorylation change measured in this study does not correlate with 


\section{A}

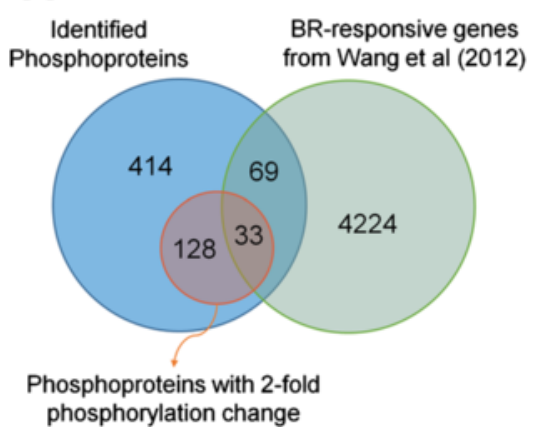

B

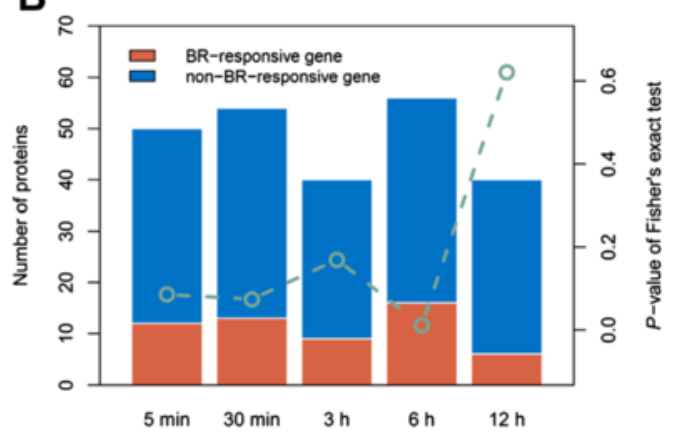

Fig. 7 Comparison between phosphorylated proteins and BR-responsive genes in transcription level. a The Venn diagram shows the overlap between phosphorylated proteins and BR-responsive genes. $\mathbf{b}$ The proportion of significantly regulated phosphoproteins which are also BR-responsive genes (red) and not BR-responsive genes (blue) at each time point is presented by bar plot. The green circle indicates the significance of the proportion of BR-responsive genes which is assessed by Fisher's exact test

transcription change in RNA level. Additionally, these also suggest that BR-signaling not only induces gene expression which regulated by BZR1 and BES1, but also change protein activation by phosphorylation via various kinases.

\section{Discussion}

BR stimulates the signaling by binding to transmembrane receptor complex to influence the activity of transcription factors, which regulate the expression of BR-responsive genes for a wild range of physiological processes. In addition to the influence of gene expression, recent studies indicated that some of the components of the BR signaling pathway involve in other signaling network to regulate diverse physiological processes $[6,7,52]$. In this study, we conducted a proteome-wide analysis of temporal phosphorylation to reveal the components of BR signaling and cross-talking pathways. A total of 1231 high confident phosphorylation sites belonging to 644 proteins were identified in three biological replicates. Of these, 239 phosphorylation sites were significantly influenced by BR that is in higher percentage than previous proteomic studies [13]. In this study, we profile the phosphorylation events responded to BR by the HAMMOC enrichment method coupling with mass spectrometry (nano LC-MS/ MS) which provides high efficiency and specificity to detect phosphopeptides [19]. It might lead to more significantly different phosphorylation sites identified in this study. In the phosphorylated residue analysis, the proportion of phosphorylation on tyrosine residues $(9 \%)$ was greater than in previous studies $[15,28,29]$, but there were no differences between our time point experiments (Fig. 2 and Table 1).

Among these identified phosphoproteins, the key components of BR signaling, including BRI1, BAK1, BKI1, BSK1, BSU1, BZR1, and BES1, were not observed in this study. We examined five recently large-scale protein phosphorylation analyses [51, 53-56], and surprisingly found most of these proteins are unable to be detected by those studies except BSK1 [53-55] and BES1 [51, 53, 54]. These results might be due to several reasons. First, the physicochemical properties of these proteins might be limited to detect their phosphorylation by current techniques. Second, BAK1 and BSK1 belong to kinases, which are low cellular abundant proteins compared with their substrates to shield theirs signals [57]. Third, previous studies showed that BR application could lead BAK1 phosphorylation within $30 \mathrm{~s}$ and reduces it after $120 \mathrm{~s}$ that might cause BAK1 not to be found in our study [58]. Finally, BRI1 and BKI1 are transmembrane proteins which are not easy to be extracted due to their hydrophobic character [59].

Several BR-signaling associated proteins such as GF14 PHI, SK 11, ATSK12, SK13, GSK1, MPK3, MPK6, BSL1, and BSL3 were detected by our phosphoproteomics experiments and PIN analysis. However, these proteins in our study did not reveal significant phosphorylation changes after BR treatment. The possible reason is that most of them might be inhibited or played as negative regulators in BR signalling [38]. Nevertheless, we still identified some BR-signaling related proteins, such as BSK3 [60] and BIL4 [49] which revealed differential phosphorylation expression after BR treatment. It indicates that our phosphoproteomics experiment can identify known BR signaling and capture potential novel BR signals.

Because the interactome of Arabidopsis is incomplete, several known BR-related proteins identified in our data were not incorporated into PIN. For example, relative of early flowering 6 (REF6) and beta-amylase 7 (BAM7) are two important regulators of BR responsive gene expression. REF6, a Jumonji domain-containing protein, interacts with BES1 to regulate target gene expression and 
coordinates BR response with other development processes [61]. BAM7, a beta-amylase-like protein, contains high similarity to the N-terminal DNA-binding domain of BZR1. Therefore, BAM7 protein can bind to the cisregulatory element similar to BZR1 but has opposite effect to BZR1 on gene expression [62].

Although only a quarter of phosphorylation sites were quantified at all five time points, our bioinformatics approaches help to reveal the dynamics of BR regulation. First, GO enrichment analysis showed the transition of biological processes after BR treatment. Then, we used Arabidopsis interactome and sequence content of phosphorylation site to connect and interpret these phosphoproteins and intended to unravel the complexity mechanisms of BR signaling (Fig. 8). In addition to BSK3, other homologs of BSK, including AT5G59010, AT3G54030, and AT5G41260, were also detected and had the consensus phosphorylation site in BSK3. Recent study demonstrates redundant biological functions for BSKs and suggests the existence of a regulatory link between BSKs and GSK3-like kinases [60]. The motif analysis results showed that the substrates of GSK3/MAPK were significantly regulated. Current research indicates that GSK3-like kinases play the crucial roles in BR signalling $[6,7]$ as well as many physiological processes were regulated by MAPK, such as stomatal development and plant immunity [63]. Therefore, the substrate of GSK3/MAPK might be considered as the downstream of BR signaling. We also compared the proteins with significantly phosphorylation changes to BRresponsive genes in transcription level and suggested that the phosphorylation difference on most of proteins observed in this study, especially at 5 and $30 \mathrm{~min}$, might be directly affected by BR signaling, not indirectly due to transcriptional regulation of BR target genes.

The constructed time-dependent kinase-substrate interaction networks may also indicate the possible mechanism of crosstalk between BR and other hormone signaling (Fig. 8). BR might activate auxin signaling by positively phosphorylating on ABCB4 which regulates cellular auxin levels, so that we observed auxin-responsive GH3-like protein (AT1G48660) was in its active form [64]. CK2 and SnRK2 are important regulators in ABA signalling $[45,65]$, and we also observed their substrates were significantly regulated at $3 \mathrm{~h}$ of BR treatment. In previous studies, their substrates, including BTR1L, RSZ32, ATRSP41, AT4G39680, and AT4G26630, were shown to have an up-regulated level after ABA induction [51]. Interestingly, these proteins have a significantly phosphorylation down-regulation level at $30 \mathrm{~min}$ in our data. It implies that although the $\mathrm{BR}$ and $\mathrm{ABA}$ interact to regulate protein phosphorylations, the molecular mechanisms seems to be complex and required more information to be clarified.
In the end, the MS-based identified proteins with significantly phosphorylation changes might result from gene or protein expression changes, therefore we compared BR-responsive genes in phosphorylation and transcription levels and found low overlap between these gene set. This result can be used as an indirect evidence to demonstrate that observed BR-responsive phosphoproteins in our quantitative phosphoproteomics analysis are not affected by transcriptional regulations. Since this result might be due to the differences of experimental conditions, we pooled all BR-responsive genes under different conditions together to eliminate the effect. However, further experimental validations are required to clarify the novel BRresponsive phosphorylation events.

\section{Conclusions}

This study provides a dynamic profile of phosphoproteome coupling bioinformatics to uncover the BRregulated networks in Arabidopsis. Phosphoproteome analysis identified 1104 unique phosphopeptides from 739 unique phosphoproteins, as well as 1231 high-confidence phosphorylation sites, of which 239 were significantly regulated after treatment with $B R$. In initial $B R$ responses, the known BR-related genes BIL4 and BSK3 with novel phosphorylation sites were identified. The motif enrichment on significantly regulated peptides reveals that BR signalling is regulated by kinases CK2, GSK3/MAPK, and SnRK2. Furthermore, these kinases play the important roles in $\mathrm{BR}$ interconnection with other plant hormone pathways, such as auxin and ABA. The phosphoproteins and phosphosites identified in our study provide a useful dataset for revealing signaling networks of BR regulation, and also expanded our knowledge of protein phosphorylation modification in plants as well as further deal to solve the plant growth problems.

\section{Methods}

\section{Plant material and growth conditions}

Plant System Biology Dark type (PSB-D) cell suspension cultures (A. thaliana ecotype Landsberg erecta) were maintained in $50 \mathrm{~mL}$ Murashige \& Skoog medium $(4.43 \mathrm{~g} / \mathrm{L}$; Duchefa Biochemie, Netherlands), $30 \mathrm{~g} / \mathrm{L}$ sucrose, $0.5 \mathrm{mg} / \mathrm{L}$ naphthalene acetic acid, $0.05 \mathrm{mg} / \mathrm{L}$ kinetin; adjusted to $\mathrm{pH} 5.7$ with $1 \mathrm{M} \mathrm{KOH}$ ) at $27{ }^{\circ} \mathrm{C}$ under gentle agitation (130 rpm) in the dark. Cells were subcultured in fresh medium at a $1 / 10$ dilution every seven days.

\section{BR treatment}

For hormone starvation of PSB-D cells, cultured medium in 7-day-old cultures was replaced with fresh medium and maintained for $48 \mathrm{~h}$, and then 24epibrassinolide (a highly active BR; Sigma-Aldrich, USA) and DMSO (mock) were added to the medium. 24epibrassinolide concentrations were $10 \mathrm{nM}, 100 \mathrm{nM}$, 


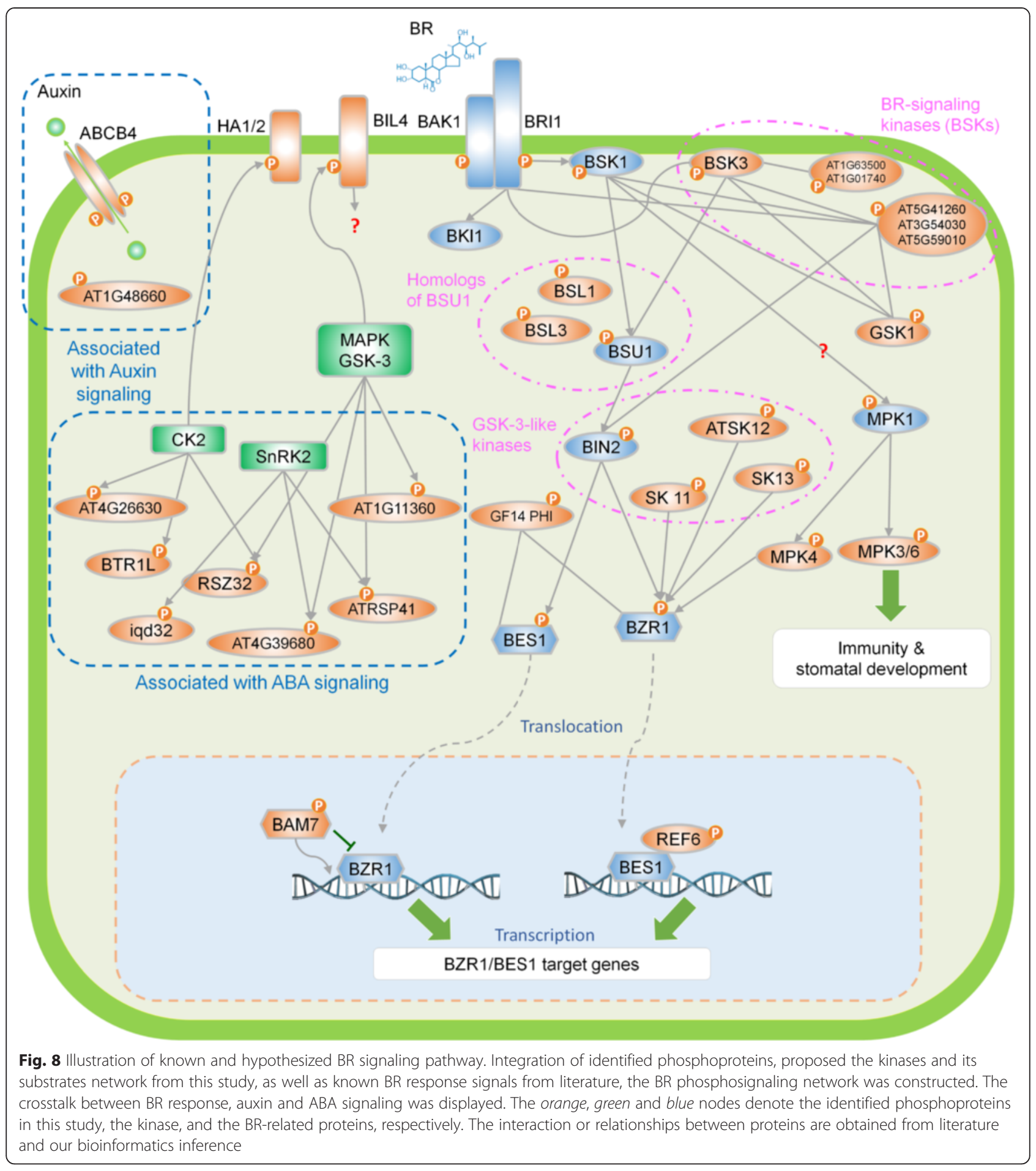

$1 \mu \mathrm{M}$, and $5 \mu \mathrm{M}$ in the gene expression assay, and $5 \mu \mathrm{M}$ in the phosphoproteome analysis. Treatment times were chosen as $3 \mathrm{~h}$ in the gene expression assay, and $5 \mathrm{~min}$, $30 \mathrm{~min}, 3 \mathrm{~h}, 6 \mathrm{~h}$, and $12 \mathrm{~h}$ in the phosphoproteome analysis. Cells $(0.2 \mathrm{~g}$, wet) were immediately washed with $0.1 \mathrm{M}$ phosphate buffer ( $\mathrm{pH}$ 5.7) and frozen in liquid nitrogen in preparation for further experiments.

\section{Real-time PCR}

Total RNA from plant cells was isolated with TRIzol Reagent (Invitrogen, USA) and cDNA was synthesized using a RevertAid $\mathrm{H}$ Minus reverse transcriptase kit (Fermentas, USA) following manufacturer's instructions. Primer design is shown in Additional file 6. Gene expressions regulated by BR were detected using an iQ5 Real- 
time PCR detection system (Bio-Rad) with a KAPA SYBR ${ }^{\circ}$ FAST One-Step qRT-PCR Bio-Rad iCycler kit (KAPA Biosystems, USA) following manufacturer's instructions.

\section{Protein extraction and trypsin digestion}

Proteins were extracted by $0.1 \mathrm{M}$ Tris- $\mathrm{HCl}(\mathrm{pH} 8.0)$ and $8 \mathrm{M}$ urea with protein phosphatase inhibitor cocktails (Bioman, Taiwan) and protease inhibitors (BioShop, Canada). Protein concentrations were quantified using a Bio-Rad Protein Assay (Bio-Rad, Munich, Germany). The protein extracts of each sample was reduced with $10 \mathrm{mM}$ dithiothreitol (Wako, Japan) for $45 \mathrm{~min}$ at room temperature, and then carbamidomethylated with $50 \mathrm{mM}$ iodoacetamide (Sigma, USA) for $45 \mathrm{~min}$ at room temperature in darkness. Alkylated proteins were diluted four times with $50 \mathrm{mM}$ triethylammonium bicarbonate (TEAB) and digested with endopeptidase Lys-C $(1: 100 \mathrm{w} / \mathrm{w})$ (Wako) for $2 \mathrm{~h}$. Subsequently, sequencinggrade modified trypsin (1:100 w/w) (Promega, Germany) was added for $16-18 \mathrm{~h}$ at room temperature. The digested peptides were acidified to a $\mathrm{pH}<3$ with trifluoroacetic acid (TFA). Acidified peptides were desalted using StageTips with SDB-XC Empore disc membranes (SDB-XC StageTip) (3 M, Germany) [66], and eluted in a buffer containing $0.1 \%$ TFA and $80 \%$ acetonitrile.

\section{Dimethylation labeling}

All peptide concentrations were quantified using the BCA protein assay (Pierce Biotechnology, Inc., Rockford, IL, USA) as per the manufacturer's instructions. $200 \mu \mathrm{g}$ tryptic peptide of each sample were dried and re-dissolved in 0.1 M TEAB. Mock-treated samples were labeled with $4 \%$ formaldehyde- $H_{2}$. BR-treated samples were labeled with formaldehyde- $D_{2}$. And then, $6 \mu \mathrm{L}$ of freshly prepared $0.6 \mathrm{M}$ sodium cyanoborohydride was immediately added to sample solution. The solution was mixed for $60 \mathrm{~min}$ at room temperature, and then $1 \%$ ammonium hydroxide was added to stop the reaction. Formic acid was used to further stop the reaction and acidify the samples. Finally, the labeled samples were combined at a 1:1 ratio and desalted using SDB-XC StageTip.

\section{Phosphopeptide enrichment}

The phosphopeptide was enriched by HAMMOC as previously described [19, 66-68]. Custom HAMMOC tips were prepared by packing $\mathrm{TiO}_{2}$ beads $(10 \mu \mathrm{m}, \mathrm{GL}$ Sciences, Japan) into C8 StageTips. Prior to sample loading, HAMMOC tips were equilibrated with solution A containing $0.1 \%$ TFA, $5 \%$ acetonitrile, and $300 \mathrm{mg} / \mathrm{mL}$ lactic acid. All peptide concentrations were quantified using the BCA protein assay (Pierce Biotechnology, Inc., Rockford, IL, USA) as per the manufacturer's instructions. About $400 \mu \mathrm{g}$ desalted peptide mixture was mixed with an equal volume of solution $\mathrm{A}$ and loaded onto the HAMMOC tips (100 $\mu$ g mixed peptides per tip). After successive washing with solution A and solution B ( $0.1 \%$ TFA and $80 \%$ acetonitrile), the resulting phosphopeptides were eluted by 0.5 and $5 \%$ piperidine (Wako). The eluent was acidified with TFA, desalted with the SDB$\mathrm{XC}$ StageTip, and vacuum-dried. The phosphopeptides were re-suspended in $0.5 \%$ TFA and subjected to nanoLC-MS/MS analysis.

\section{NanoLC-MS/MS analysis}

For mass spectrometry analysis, LC was performed on an Agilent 1100 series HPLC system (Agilent Technologies, USA) with a micro-T for flow splitting coupled to an LTQ-Orbitrap XL hybrid mass spectrometer (Thermo Electron, USA) equipped with a PicoView nanospray interface (New Objective, USA). Peptide mixtures were loaded onto a $75 \mu \mathrm{m} \times 250 \mathrm{~mm}$ fused silica capillary column packed in-house with $\mathrm{C} 18$ resin $(5 \mu \mathrm{m}$, Nucleosil 120-5 C18; Macherey-Nagel, Germany), and were separated over 110 min using a linear gradient of $2-50 \%$ solvent B (95\% acetonitrile with $0.1 \%$ formic acid) and solvent A ( $0.1 \%$ formic acid in water) at a flow rate of $300 \mathrm{~nL} / \mathrm{min}$. The LTQ-Orbitrap was operated in positive ion mode and mass spectra from full scans were acquired on the Orbitrap analyzer $(\mathrm{m} / \mathrm{z} 350-1600)$ with resolution set to $R=30,000$ (at $\mathrm{m} / \mathrm{z} 400$ ). The ten most intense peptide ions were selected from the MS scan and fragmented by collision-induced dissociation for MS/MS scan in the LTQ analyzer. All Orbitrap measurements were performed with the "lock mass" option to improve mass accuracy of precursor ions.

\section{Data analysis and database search}

Raw spectrum files were processed for phosphopeptide identification and phosphosite quantification with MaxQuant software version 1.3.0.5 (http://maxquant. org/) [69]. Peptide identification was performed using Andromeda search engine [70] against the Arabidopsis TAIR10 database (http://www.arabidopsis.org/; version pep_20101214). Search criteria were trypsin specificity, fixed modification of carbamidomethyl, variable modifications of oxidationand phosphorylation, and two allowed missed cleavages. A minimum peptide length of seven amino acids was required. Precursor mass tolerance was set at $6 \mathrm{ppm}$, and fragment ion tolerance at $0.5 \mathrm{Da}$. A target-decoy search strategy was used in this study [71]. Protein, peptide, and site identification were accepted on the basis of posterior error probability with a false discovery rate (FDR) of $1 \%$. Precursors of already identified peptides were further searched using the "match between runs" option in MaxQuant, which matches precursor masses in a 2 min retention time window. The localization probability of all putative phosphorylation 
sites was determined using the MaxQuant posttranslational modification score algorithm. The proteomics data have been deposited to the ProteomeXchange Consortium [72] via the PRIDE partner repository with the dataset identifier PXD001473. Proteins were counted separately if a peptide matched to multiple proteins in the database search. Identified phosphorylation sites were grouped into three classes based on the phosphorylation localization probability score: class I $(p>$ $0.75)$, class II $(0.5<p \leq 0.75)$, and class III $(p \leq 0.5)$ [73]. Class I sites were considered as confident assignments and used for all analyses in the study.

\section{Construction of protein-protein interaction network}

We collected protein-protein interactions of Arabidopsis from BioGRID [35],AtPID [36], and PhosPhAt [37]. Additional, the known BR signaling components were collected from literature $[3,6,7]$. The interactions which connect the two identified phosphoproteins or link identified phosphoproteins to the known BR-related proteins were assembled into a network. The constructed network was visualized by Cytoscape [74].

\section{Gene ontology enrichment analyses}

The Gene Ontology (GO) annotation for all Arabidopsis proteins was obtained from TAIR [75]. The onesided Fisher's exact tests were performed to identify the over-representative GO terms. P-values returned by one-sided Fisher's exact tests were adjusted via the Benjamini-Hochberg method to control false discovery rate (FDR). The enrichment analysis for biological process (BP) and cellular component (CC) ontologies were done separately for each of time points.

For further hierarchical clustering based on BP GO terms, we first collated all the GO terms obtained after the enrichment, and the filtered for those GO terms which were at least enriched in one the of time points with adjusted $P$-value $<0.05$. This filtered $P$-value matrix was transformed by the function $x=-\log _{10}$ ( $P$-value). For each GO term, the mean $(\mu)$ and standard deviation $(\rho)$ of these $x$ values were calculated, and then $x$ values were $\mathrm{z}$-transformed by following formula:

$$
z=\frac{x-\mu}{\rho}
$$

These $\mathrm{z}$ scores were then clustered by one-way hierarchical clustering by Pearson correlation coefficient and average linkage clustering in GAP (Generalized Association Plots) [76].

\section{Phosphorylation motif analysis}

All confident phosphopeptide sequences were submitted to Motif-X (v1.2) [77] for the identification of over- represented motifs. Sequences were centered on each phosphorylation site and extended to 15 amino acids ( \pm 7 residues). Where the phosphorylation site was close to the N/C-terminal of the protein, the sequence was filled with up to 15 amino acids with the required number of " $X$ " which denotes any amino acid. The significance threshold was set to $P<10^{-6}$ and the occurrence threshold, the minimum number of times you wish each of your extracted motifs to occur in the data set, was set to 30 for Ser and 20 for Thr and Tyr. The Arabidopsis International Protein Index (IPI) database provided by Motif-X was used as a background. Over-represented motifs were associated with specific kinases by searching manually the literature and databases, including PhosPhAt (the Arabidopsis phosphorylation site database) [37], HPRD (Human Protein Reference Database) [42], and PhosphoNetworks [43]. A motif-kinase association was constructed if the motif was identical to the recognizing pattern of a given kinase reported by literature or described in a database.

To reveal the dynamics of kinase regulations, we assessed the situation of each enriched motif at each time point. For a motif $m$ at the time point $t$, one sideFisher's exact test was performed using the $2 \times 2$ contingency table that include the following numbers: $k$, $K-k, n, N-n$ where $k$ denotes the number of peptides which intensity ratios at $t$ pass a threshold and contain the motif $m, K$ is the total number of peptides which intensity ratios at $t$ pass a threshold (i.e. $\mid \log _{2}$ (foldchange) $\mid \geqq 1$ ), $n$ denotes the number of peptides which are identified at $t$ and contain motif $m$, and $N$ is the total number of peptides identified at $t$. We analyzed separately the up- and down-regulated peptides.

\section{Comparison between differential phosphorylation proteins and expression genes of BR-responsive}

The BR-responsive genes derived from genome-wide transcriptional experiments were obtained from Wang and colleagues [6]. The overlap between differential phosphorylation protein set and differential expression gene set was assessed by Fisher's exact test.

\section{Additional files}

Additional file 1: The list of all quantified phosphorylation sites.

Additional file 2: The distribution of phosphorylation sites with missing quantification.

Additional file 3: Biological process ontology enrichment analysis at each time point.

Additional file 4: Arabidopsis consensus motifs correspond to human kinases).

Additional file 5: Identified phosphoproteins also involving in expression regulation by BR induction.

Additional file 6: Primer list in this study. 


\section{Abbreviations}

ABA: Abscisic acid; ABCB4: ATP-binding cassette sub-family B member 4 AtPID: Arabidopsis thaliana Protein Interactome Database;

ATSK12: Shaggy-related kinase 12; BAK1: Bri1-associated receptor kinase 1; BAM7: Beta-amylase 7; BES1: Bri1-ems-suppressor 1; BIL4: Brz-insensitive-long hypocotyls 4; BIN2: Br-insensitive 2; BKI1: Bri1 kinase inhibitor 1; BP: Biological process; BR: Brassinosteroid; BRI1: BR-insensitive 1; BRU6: Indole-3-acetic acid amidosynthetase; BSK: BR-signaling kinase; BSU1: Bri1 suppressor1; BZR1: Brassinazole-resistant 1; CC: Cellular component; CK: Mock; CSNK1G3: Casein kinase 1, gamma 3;

DYRK4: Dual-specificity tyrosine-phosphorylation regulated kinase 4; FDR: False discovery rate; FLS2: Flagellin-sensing 2; GAP: Generalized Association Plots; GF14 PHI: 14-3-3-like protein G-box factor 14 phi; GO: Gene Ontology; GSK1: Shaggy-like protein kinase 1; GSK3: Glycogen synthases kinase 3; HAs: H(+)-ATPases; HAMMOC: Aliphatic hydroxy acid-modified metal oxide chromatography; HPRD: Human Protein Reference Database; IMAC: Immobilized metal-affinity chromatography; lad32: IQ-domain 32; LAST1: Large tumor suppressor kinase 1; MAPK: Mitogen activated protein kinase; MKK4/5/7/9: Mitogen-activated protein kinase kinases 4, 5, 7 and 9; MPK3/6: Mitogen-activated protein kinase 3 and 6; NanoLC-MS/MS: Nanoliquid chromatography-tandem mass spectrometry; NEK2: NIMA-related kinase 2; PDIK1L: PDLIM1 interacting kinase 1 like; PhosPhAt: Arabidopsis phosphorylation site database; PIN: Protein-protein interaction network; PRPF4B: Pre-mRNA processing factor 4B; PSB-D: Plant System Biology-Dark type culture; REF6: Relative of early flowering 6; RLCK: Receptor-like cytoplasmic kinases; SAUR-AC1: Small auxin up RNA; SCX: Strong cation exchange; SK 11: Shaggy-related kinase 11; SK13: Shaggy-related kinase 13; SnRK2: SNF1-related kinase II; TAIR: The Arabidopsis Information Resource; TEAB: Triethylammonium bicarbonate; TFA: Trifluoroacetic acid; TiO2: Titanium dioxide; YODA: Mitogen-activated protein kinase kinase kinase YODA.

\section{Competing interests}

The authors declare that they have no competing interests.

\section{Authors' contributions}

$\mathrm{HCH}$ and HFJ conceived and designed the study. LLL, CWH and SYK performed cell culture, real-time PCR, phosphoproteomics experiments, and peptide identification. CLH performed bioinformatics analysis. LLL, CLH and HFJ interpreted the data and wrote the manuscript. HCH and HFJ supervised the study. HLH provide PSB-D cells. All authors read and approved the final manuscript.

\section{Acknowledgements}

We thank the Institute of Biological Chemistry, Academic Sinica for mass spectrometric analysis and Divya Sahu for proofreading the manuscript. This work was supported by the National Taiwan University Cutting-Edge Steering Research Project (NTU-CESRP-103R7602C3), the National Science Council of Taiwan (NSC 102-2628-B-002-041-MY3, NSC 102-2627-B-002-002, NSC 102-2311-B-010-004) and Ministry of Science and Technology (MOST 103-2320-B-010-031-MY3).

\section{Author details}

${ }^{1}$ Department of Life Science, National Taiwan University, No. 1, Sec. 4, Roosevelt Road, Taipei 106, Taiwan. ${ }^{2}$ Institute of Molecular and Cellular Biology, National Taiwan University, No. 1, Sec. 4, Roosevelt Road, Taipei 106, Taiwan. ${ }^{3}$ Institute of Plant Biology, National Taiwan University, No. 1, Sec. 4, Roosevelt Road, Taipei 106, Taiwan. ${ }^{4}$ Institute of Biomedical Informatics, Center for Systems and Synthetic Biology, National Yang-Ming University, No.155, Sec.2, Linong Street, Taipei 112, Taiwan. ${ }^{5}$ Graduate Institute of Biomedical Electronic and Bioinformatics, National Taiwan University, No. 1, Sec. 4, Roosevelt Road, Taipei 106, Taiwan.

\section{Received: 2 November 2014 Accepted: 6 July 2015}

Published online: 18 July 2015

\section{References}

1. Clouse SD, Langford M, McMorris TC. A brassinosteroid-insensitive mutant in Arabidopsis thaliana exhibits multiple defects in growth and development. Plant Physiol. 1996;111(3):671-8.
2. Clouse SD, Sasse JM. BRASSINOSTEROIDS: essential regulators of plant growth and development. Annu Rev Plant Physiol Plant Mol Biol. 1998:49:427-51.

3. Oh MH, Wang X, Clouse SD, Huber SC. Deactivation of the Arabidopsis BRASSINOSTEROID INSENSITIVE 1 (BRI1) receptor kinase by autophosphorylation within the glycine-rich loop. Proc Natl Acad Sci U S A. 2012;109(1):327-32.

4. Sharma P, Bhardwaj R. Effects of 24-epibrassinolide on growth and metal uptake in Brassica juncea L. under copper metal stress. Acta Physiol Plant. 2007;29(3):259-63.

5. Sharma P, Bhardwaj R, Arora N, Arora HK, Kumar A. Effects of 28-homobrassinolide on nickel uptake, protein content and antioxidative defence system in Brassica juncea. Biol Plant. 2008;52(4):767-70.

6. Wang ZY, Bai MY, Oh E, Zhu JY. Brassinosteroid signaling network and regulation of photomorphogenesis. Annu Rev Genet. 2012;46:701-24.

7. Kim TW, Wang ZY. Brassinosteroid signal transduction from receptor kinases to transcription factors. Annu Rev Plant Biol. 2010;61:681-704.

8. Vert G, Chory J. Downstream nuclear events in brassinosteroid signalling. Nature. 2006;441(7089):96-100.

9. Kim TW, Michniewicz M, Bergmann DC, Wang ZY. Brassinosteroid regulates stomatal development by GSK3-mediated inhibition of a MAPK pathway. Nature. 2012:482(7385):419-22.

10. Albrecht C, Boutrot F, Segonzac C, Schwessinger B, Gimenez-Ibanez S, Chinchilla D, et al. Brassinosteroids inhibit pathogen-associated molecular pattern-triggered immune signaling independent of the receptor kinase BAK1. Proc Natl Acad Sci U S A. 2012;109(1):303-8.

11. Huang B, Chu CH, Chen SL, Juan HF, Chen YM. A proteomics study of the mung bean epicotyl regulated by brassinosteroids under conditions of chilling stress. Cell Mol Biol Lett. 2006;11(2):264-78.

12. Deng Z, Zhang X, Tang W, Oses-Prieto JA, Suzuki N, Gendron JM, et al. A proteomics study of brassinosteroid response in Arabidopsis. Mol Cell Proteomics. 2007;6(12):2058-71.

13. Tang W, Deng Z, Oses-Prieto JA, Suzuki N, Zhu S, Zhang X, et al. Proteomics studies of brassinosteroid signal transduction using prefractionation and two-dimensional DIGE. Mol Cell Proteomics. 2008;7(4):728-38.

14. Shigeta T, Yasuda D, Mori T, Yoshimitsu Y, Nakamura Y, Yoshida S, et al. Characterization of brassinosteroid-regulated proteins in a nuclear-enriched fraction of Arabidopsis suspension-cultured cells. Plant Physiol Biochem. 2011:49(9):985-95.

15. Nakagami H, Sugiyama N, Mochida K, Daudi A, Yoshida Y, Toyoda T, et al. Large-scale comparative phosphoproteomics identifies conserved phosphorylation sites in plants. Plant Physiol. 2010;153(3):1161-74.

16. Hojlund K, Bowen BP, Hwang H, Flynn CR, Madireddy L, Geetha T, et al. In vivo phosphoproteome of human skeletal muscle revealed by phosphopeptide enrichment and HPLC-ESI-MS/MS.J Proteome Res. 2009;8(11):4954-65.

17. Fila J, Honys D. Enrichment techniques employed in phosphoproteomics. Amino Acids. 2012;43(3):1025-47.

18. Salih E. Phosphoproteomics by mass spectrometry and classical protein chemistry approaches. Mass Spectrom Rev. 2005;24(6):828-46.

19. Sugiyama N, Masuda T, Shinoda K, Nakamura A, Tomita M, Ishihama Y. Phosphopeptide enrichment by aliphatic hydroxy acid-modified metal oxide chromatography for nano-LC-MS/MS in proteomics applications. Mol Cell Proteomics. 2007;6(6):1103-9.

20. Hashimoto M, Komatsu S. Proteomic analysis of rice seedlings during cold stress. Proteomics. 2007;7(8):1293-302.

21. Ndimba BK, Chivasa S, Hamilton JM, Simon WJ, Slabas AR. Proteomic analysis of changes in the extracellular matrix of Arabidopsis cell suspension cultures induced by fungal elicitors. Proteomics. 2003;3(6):1047-59.

22. Pang $C Y$, Wang $H$, Pang $Y, X u C$, Jiao $Y$, Qin $Y M$, et al. Comparative proteomics indicates that biosynthesis of pectic precursors is important for cotton fiber and Arabidopsis root hair elongation. Mol Cell Proteomics. 2010;9(9):2019-33.

23. Bell AW, Deutsch EW, Au CE, Kearney RE, Beavis R, Sechi S, et al. A HUPO test sample study reveals common problems in mass spectrometry-based proteomics. Nat Methods. 2009:6(6):423-30.

24. White MY, Brown DA, Sheng S, Cole RN, O'Rourke B, Van Eyk JE. Parallel proteomics to improve coverage and confidence in the partially annotated Oryctolagus cuniculus mitochondrial proteome. Mol Cell Proteomics. 2011;10(2):M110 00429.

25. de Godoy LM, Olsen JV, Cox J, Nielsen ML, Hubner NC, Frohlich F, et al. Comprehensive mass-spectrometry-based proteome quantification of haploid versus diploid yeast. Nature. 2008;455(7217):1251-4 
26. Schrimpf SP, Weiss M, Reiter L, Ahrens $\mathrm{CH}$, Jovanovic M, Malmstrom J, et al. Comparative functional analysis of the Caenorhabditis elegans and Drosophila melanogaster proteomes. PLoS Biol. 2009;7(3), e48.

27. Goh WW, Lee $\mathrm{YH}$, Chung M, Wong L. How advancement in biological network analysis methods empowers proteomics. Proteomics. 2012;12(4-5):550-63.

28. Sugiyama N, Nakagami H, Mochida K, Daudi A, Tomita M, Shirasu K, et al. Large-scale phosphorylation mapping reveals the extent of tyrosine phosphorylation in Arabidopsis. Mol Syst Biol. 2008:4:193.

29. Reiland S, Messerli G, Baerenfaller K, Gerrits B, Endler A, Grossmann J, et al. Large-scale Arabidopsis phosphoproteome profiling reveals novel chloroplast kinase substrates and phosphorylation networks. Plant Physiol. 2009;150(2):889-903.

30. Goda H, Shimada Y, Asami T, Fujioka S, Yoshida S. Microarray analysis of brassinosteroid-regulated genes in Arabidopsis. Plant Physiol. 2002;130(3):1319-34

31. Hu YX, Wang YX, Liu XF, Li JY. Arabidopsis RAV1 is down-regulated by brassinosteroid and may act as a negative regulator during plant development. Cell Res. 2004;14(1):8-15.

32. Xie L, Yang C, Wang $X$. Brassinosteroids can regulate cellulose biosynthesis by controlling the expression of CESA genes in Arabidopsis. J Exp Bot. 2011;62(13):4495-506.

33. Sun Y, Fan XY, Cao DM, Tang W, He K, Zhu JY, et al. Integration of brassinosteroid signal transduction with the transcription network for plant growth regulation in Arabidopsis. Dev Cell. 2010;19(5):765-77.

34. De Rybel B, Audenaert D, Vert G, Rozhon W, Mayerhofer J, Peelman F, et al. Chemical inhibition of a subset of Arabidopsis thaliana GSK3-like kinases activates brassinosteroid signaling. Chem Biol. 2009;16(6):594-604.

35. Chatr-Aryamontri A, Breitkreutz BJ, Heinicke S, Boucher L, Winter A, Stark C, et al. The BioGRID interaction database: 2013 update. Nucleic Acids Res. 2013;41(Database issue):D816-23.

36. Li P, Zang W, Li Y, Xu F, Wang J, Shi T. AtPID: the overall hierarchical functional protein interaction network interface and analytic platform for Arabidopsis. Nucleic Acids Res. 2011;39(Database issue):D1130-3.

37. Zulawski M, Braginets R, Schulze WX. PhosPhAt goes kinases-searchable protein kinase target information in the plant phosphorylation site database PhosPhAt. Nucleic Acids Res. 2013;41(Database issue):D1176-84.

38. Kim TW, Guan S, Sun Y, Deng Z, Tang W, Shang JX, et al. Brassinosteroid signal transduction from cell-surface receptor kinases to nuclear transcription factors. Nat Cell Biol. 2009;11(10):1254-60.

39. Casson SA, Hetherington AM. GSK3-like kinases integrate brassinosteroid signaling and stomatal development. Sci Signal. 2012;5(233), e30.

40. Khan M, Rozhon W, Bigeard J, Pflieger D, Husar S, Pitzschke A, et al. Brassinosteroid-regulated GSK3/Shaggy-like kinases phosphorylate mitogen-activated protein (MAP) kinase kinases, which control stomata development in Arabidopsis thaliana. J Biol Chem. 2013;288(11):7519-27.

41. Gampala SS, Kim TW, He JX, Tang W, Deng Z, Bai MY, et al. An essential role for 14-3-3 proteins in brassinosteroid signal transduction in Arabidopsis. Dev Cell. 2007;13(2):177-89.

42. Amanchy R, Periaswamy B, Mathivanan S, Reddy R, Tattikota SG, Pandey A. A curated compendium of phosphorylation motifs. Nat Biotechnol. 2007;25(3):285-6.

43. Hu J, Rho HS, Newman RH, Zhang J, Zhu H, Qian J. PhosphoNetworks: a database for human phosphorylation networks. Bioinformatics. 2014;30(1):141-2.

44. Marques-Bueno MM, Moreno-Romero J, Abas L, De Michele R, Martinez MC. A dominant negative mutant of protein kinase CK2 exhibits altered auxin responses in Arabidopsis. Plant J. 2011;67(1):169-80.

45. Riera M, Figueras M, Lopez C, Goday A, Pages M. Protein kinase CK2 modulates developmental functions of the abscisic acid responsive protein Rab17 from maize. Proc Natl Acad Sci U S A. 2004;101(26):9879-84.

46. Zhang S, Cai Z, Wang X. The primary signaling outputs of brassinosteroids are regulated by abscisic acid signaling. Proc Natl Acad Sci U S A. 2009;106(11):4543-8.

47. Xue L, Wang P, Wang L, Renzi E, Radivojac P, Tang H, et al. Quantitative measurement of phosphoproteome response to osmotic stress in arabidopsis based on Library-Assisted eXtracted Ion Chromatogram (LAXIC). Mol Cell Proteomics. 2013;12(8):2354-69.

48. Witthoft J, Caesar K, Elgass K, Huppenberger P, Kilian J, Schleifenbaum F, et al. The activation of the Arabidopsis P-ATPase 1 by the brassinosteroid receptor BRI1 is independent of threonine 948 phosphorylation. Plant Signal Behav. 2011;6(7):1063-6.

49. Yamagami A, Nakazawa M, Matsui M, Tujimoto M, Sakuta M, Asami T, et al. Chemical genetics reveal the novel transmembrane protein BIL4, which mediates plant cell elongation in brassinosteroid signaling. Biosci Biotechnol Biochem. 2009:73(2):415-21.

50. Abel S, Burstenbinder K, Muller J. The emerging function of IQD proteins as scaffolds in cellular signaling and trafficking. Plant Signal Behav. 2013;8(6), e24369.

51. Wang P, Xue L, Batelli G, Lee S, Hou YJ, Van Oosten MJ, et al. Quantitative phosphoproteomics identifies SnRK2 protein kinase substrates and reveals the effectors of abscisic acid action. Proc Natl Acad Sci U S A. 2013;110(27):11205-10

52. Clouse SD. Brassinosteroid signal transduction: from receptor kinase activation to transcriptional networks regulating plant development. Plant Cell. 2011;23(4):1219-30.

53. Wang X, Bian Y, Cheng K, Gu LF, Ye M, Zou H, et al. A large-scale protein phosphorylation analysis reveals novel phosphorylation motifs and phosphoregulatory networks in Arabidopsis. J Proteomics. 2013;78:486-98.

54. Umezawa T, Sugiyama N, Takahashi F, Anderson JC, Ishihama Y, Peck SC, et al. Genetics and phosphoproteomics reveal a protein phosphorylation network in the abscisic acid signaling pathway in Arabidopsis thaliana. Sci Signal. 2013;6(270):rs8.

55. Engelsberger WR, Schulze WX. Nitrate and ammonium lead to distinct global dynamic phosphorylation patterns when resupplied to nitrogen-starved Arabidopsis seedlings. Plant J. 2012;69(6):978-95.

56. Mayank P, Grossman J, Wuest S, Boisson-Dernier A, Roschitzki B, Nanni P, et al. Characterization of the phosphoproteome of mature Arabidopsis pollen. Plant J. 2012;72(1):89-101.

57. Daub H, Olsen JV, Bairlein M, Gnad F, Oppermann FS, Korner R, et al. Kinase-selective enrichment enables quantitative phosphoproteomics of the kinome across the cell cycle. Mol Cell. 2008;31(3):438-48.

58. Schulze B, Mentzel T, Jehle AK, Mueller K, Beeler S, Boller T, et al. Rapid heteromerization and phosphorylation of ligand-activated plant transmembrane receptors and their associated kinase BAK1. J Biol Chem. 2010;285(13):9444-51.

59. Santoni V. Plant plasma membrane protein extraction and solubilization for proteomic analysis. Methods Mol Biol. 2007;355:93-109.

60. Sreeramulu S, Mostizky Y, Sunitha S, Shani E, Nahum H, Salomon D, et al. BSKs are partially redundant positive regulators of brassinosteroid signaling in Arabidopsis. Plant J. 2013;74(6):905-19.

61. Yu X, Li L, Li L, Guo M, Chory J, Yin Y. Modulation of brassinosteroid-regulated gene expression by Jumonji domain-containing proteins ELF6 and REF6 in Arabidopsis. Proc Natl Acad Sci U S A. 2008;105(21):7618-23.

62. Reinhold H, Soyk S, Simkova K, Hostettler C, Marafino J, Mainiero S, et al. Beta-amylase-like proteins function as transcription factors in Arabidopsis, controlling shoot growth and development. Plant Cell. 2011;23(4):1391-403.

63. Kong X, Pan J, Cai G, Li D. Recent insights into brassinosteroid signaling in plants: its dual control of plant immunity and stomatal development. Mol Plant. 2012;5(6):1179-81.

64. Kubes M, Yang H, Richter GL, Cheng Y, Mlodzinska E, Wang X, et al. The Arabidopsis concentration-dependent influx/efflux transporter $A B C B 4$ regulates cellular auxin levels in the root epidermis. Plant J. 2012;69(4):640-54.

65. Kulik A, Wawer I, Krzywinska E, Bucholc M, Dobrowolska G. SnRK2 protein kinases-key regulators of plant response to abiotic stresses. OMICS. 2011;15(12):859-72.

66. Rappsilber J, Mann M, Ishihama Y. Protocol for micro-purification, enrichment, pre-fractionation and storage of peptides for proteomics using StageTips. Nat Protoc. 2007;2(8):1896-906.

67. Ravichandran A, Sugiyama N, Tomita M, Swarup S, Ishihama Y. Ser/Thr/Tyr phosphoproteome analysis of pathogenic and non-pathogenic Pseudomonas species. Proteomics. 2009;9(10):2764-75.

68. Kyono Y, Sugiyama N, Tomita M, Ishihama Y. Chemical dephosphorylation for identification of multiply phosphorylated peptides and phosphorylation site determination. Rapid Commun Mass Spectrom. 2010;24(15):2277-82.

69. Cox J, Mann M. MaxQuant enables high peptide identification rates, individualized p.p.b.-range mass accuracies and proteome-wide protein quantification. Nat Biotechnol. 2008;26(12):1367-72.

70. Cox J, Neuhauser N, Michalski A, Scheltema RA, Olsen JV, Mann M. Andromeda: a peptide search engine integrated into the MaxQuant environment. J Proteome Res. 2011;10(4):1794-805.

71. Elias JE, Gygi SP. Target-decoy search strategy for increased confidence in large-scale protein identifications by mass spectrometry. Nat Methods. 2007;4(3):207-14. 
72. Samakovli D, Margaritopoulou T, Prassinos C, Milioni D, Hatzopoulos P. Brassinosteroid nuclear signaling recruits HSP90 activity. New Phytol. 2014;203(3):743-57.

73. Olsen JV, Blagoev B, Gnad F, Macek B, Kumar C, Mortensen P, et al. Global, in vivo, and site-specific phosphorylation dynamics in signaling networks. Cell. 2006;127(3):635-48.

74. Smoot ME, Ono K, Ruscheinski J, Wang PL, Ideker T. Cytoscape 2.8: new features for data integration and network visualization. Bioinformatics. 2011;27(3):431-2.

75. Lamesch P, Berardini TZ, Li D, Swarbreck D, Wilks C, Sasidharan R, et al. The Arabidopsis Information Resource (TAIR): improved gene annotation and new tools. Nucleic Acids Res. 2012;40(Database issue):D1202-10.

76. Wu HM, Tien YJ, Chen CH. GAP: a graphical environment for matrix visualization and cluster analysis. Comput Stat Data An. 2010;54(3):767-78.

77. Schwartz D, Gygi SP. An iterative statistical approach to the identification of protein phosphorylation motifs from large-scale data sets. Nat Biotechnol. 2005;23(11):1391-8.

\section{Submit your next manuscript to BioMed Central and take full advantage of:}

- Convenient online submission

- Thorough peer review

- No space constraints or color figure charges

- Immediate publication on acceptance

- Inclusion in PubMed, CAS, Scopus and Google Scholar

- Research which is freely available for redistribution 